\title{
Effect of double embryo transfer derived from autologous frozen oocytes on multiple pregnancy rates and presentation of success rates stratified by age at retrieval
}

\author{
(D) Ahmad Badeghiesh, (D) Rea Konci, (D) Sarah Aldhaheri, (D) Weon-Young Son, (D) Michael H. Dahan \\ Department of Obstetrics and Gynecology, McGill University Health Center, Montreal, Canada
}

\section{Abstract}

Objective: To compare outcomes transferring one or two embryos in autologous frozen oocyte cycles.

Material and Methods: A retrospective cohort study conducted at an academic fertility center between January 2012 and December 2018. One-hundred and fourteen patients underwent frozen oocyte transfers; 67 single embryo transfer (SET) and 47 double embryo transfer (DET). No subjects had more than two embryos transferred. Data were analyzed using t-test and chi-squared testing. Multivariate logistic regression was used to control for confounding effects. Power analysis suggested an $82 \%$ power with alpha of $5 \%$ and effect size of $27 \%$.

Results: Regarding the embryo stage, $72 \%$ were cleavage embryos and $28 \%$ were blastocyst embryos. Among those who had cleavage stage embryos, $48.8 \%$ underwent SET and $51.2 \%$ underwent DET. In the blastocyst embryos group these proportions were $84.4 \%$ and $15.6 \%$, respectively. There were no difference in pregnancy rate for SET (40.3\%) vs DET (36.2\%) ( $p=0.78)$. Additionally, the live birth rate did not differ between SET and DET ( 28.4 vs $19.1 \%$, respectively, $\mathrm{p}=0.26)$. The multivariate multilevel analysis provided adjusted odds ratios ( $95 \%$ confidence interval) of: 1.85 (0.46-7.44) for pregnancy; 0.497 (0.05-4.86) for clinical pregnancy; and 0.82 (0.11-6.29) for live birth when comparing SET and DET. Multiple pregnancy rates were significantly lower in the SET (0\%), compared with DET group (44.4\%) $(\mathrm{p}<0.002)$.

Conclusion: SET results in excellent live birth outcomes in autologous frozen oocyte cycles. However DET results in significantly increased rates of multiple pregnancies. This suggests that SET is a viable option in autologous frozen oocyte cycles. (J Turk Ger Gynecol Assoc 2021; 22: 168-73)

Keywords: Oocyte vitrification, single embryo transfer, autologus, multiple pregnancy rates

Received: 06 November, 2020 Accepted: 29 April, 2021

\section{Introduction}

In the early days of in vitro fertilization (IVF) multiple embryos were often transferred in order to maximize the chances of a successful pregnancy but this number has gradually decreased over time to limit the complications associated with multiple pregnancy (1-3). The American Society for Reproductive Medicine and the Society for Assisted Reproductive Technology (SART) developed guidelines to limit the number of embryos transferred in order to decrease the number of multiple gestations as a result of reproductive technologies (2). Keeping in mind that the ultimate goal of IVF is a healthy, singleton, live birth, recommendations are made regarding the number of embryos to be transferred based on particular characteristics, including patient age and stage of embryo being transferred. For women under the age of 35 years undergoing IVF, the transfer of a single embryo, regardless of embryo stage, is the generally accepted practice, though two embryos may be considered if the prognosis of pregnancy after the transfer is lower. For women between the ages of 35-37 years, a consideration should be made for a single-embryo transfer. For women between 38-40 years old, SART recommended not more than three 
cleavage-stage embryos or two blastocyst embryos should be transferred. For patients who have euploid embryos available after pre-implantation genetic testing for aneuploidy (PGT-A), the transfer of one embryo should be the practice norm. In the 41 to 42 year-old age group, there should be no more than four cleavage-stage embryos or three blastocysts transferred. Similarly, if euploid embryos are available, the norm should the transfer of a single blastocyst.

Significant efforts have been made to reduce the incidence of higher order births such as triplets and quadruplets, but twin pregnancy rates have not seen the same decline as a result of double embryo transfers (DET), which continues to be commonly practiced (4). It should be noted that the incidence of monozygotic twins in IVF pregnancies is in fact higher than the incidence in spontaneous pregnancies, a factor that is out of the provider's control (5).

A recent study by Freeman et al. (6) found that the transfer of a single vitrified-warmed blastocyst is an acceptable practice, given that it maintains live birth rates and decreases the rate of multiple pregnancies and its complications in patients younger than 38 years old. It was previously demonstrated that transferring more than one blastocyst in women 40 years of age and greater using autologous gametes, increases the multiple pregnancy rate without significantly changing the live birth rate $(7,8)$. Cumulative live birth rates are maximized when transferring serial single blastocysts in women 40 years of age and greater (9). An advance in reproductive technologies was made with the introduction of vitrification. Vitrification is when the gamete or embryo undergoes dehydration followed by ultra-rapid cooling which causes minimal ice crystal formation and little if any damage. The development of vitrification allowed the expansion of the oocyte freezing industry. Until the introduction of vitrification, oocytes did not freeze well and usually did not survive once thawed or failed to be competent to develop into an embryo with high live birth potential. Women have increasingly used oocyte freezing to maintain fertility potential later in life. However, few centers have extensive knowledge of returning embryos from these frozen oocytes. As oocytes have previously demonstrated greater likelihood of suffering freezing damage than embryos, the role of single embryo transfer in this group has been minimally studied. Although we believe oocytes that have been vitrified provide excellent pregnancy potential, few large studies verify this. However, a study demonstrated that single embryo transfer (SET) after oocyte vitrification can lead to excellent pregnancy rates (6). This study failed to stratify for age of the patient at oocyte collection and did not include older women. The goal of our study was to compare SET with DET during autologous frozen oocyte cycles using live birth, pregnancy rates, and multiple pregnancy rates as our primary outcomes in women of all ages, stratified by age at oocyte collection.

\section{Material and Methods}

\section{Study design}

This is a retrospective cohort study conducted at a single academic fertility center between January 2012 and December 2018, including patients who underwent frozen embryo transfers. The patients were sub-divided into those undergoing SET and those undergoing DET. None of the patients received more than two embryo transfers during the time period of the study. This is the current limit based on government regulation in our jurisdiction. All included patients had a normal uterine cavity, no hydrosalpinx, and no thyroid or prolactin abnormalities.

McGill University Health Center Institutional Review Board (IRB) Ethics approval of this retrospective study was obtained (approval number: 2020- 5631).

The risks and benefits of SET vs DET were discussed between the clinical staff and the patients. The risks associated with multiple gestation pregnancy for both the mother and the fetus were also thoroughly discussed. Women under the age of 37 years at the time of embryo transfer must receive a single embryo while women 37 years of age or more could receive a maximum of two embryos. This permitted women who froze oocytes at age 30 and were now undergoing embryo transfer at the age of 37 years old or older to be eligible for the transfer of two embryos. Up until 2016 there was a limit of transfer for two embryos in women under 37 years of age. At the beginning of 2016 this limit was reduced to one embryo.

Exclusion criteria included untreated uterine leiomyoma, polyps, or hydrosalpinx, untreated thyroid or prolactin abnormalities, women who did not undergo transfer of embryos developed from oocyte vitrification and those who had experienced previous failed embryo transfers. All subjects were included only once.

\section{Ovarian stimulation and egg retrieval}

Women underwent gonadotropin stimulation with a combination of follicle stimulating hormone and human menopausal gonadotropin as part of a gonadotropin-releasing hormone agonist long protocol or antagonist cycle. These cycles have been previously reported in detail by our group (10).

A subcutaneous injection of either recombinant human chorionic gonadotropin (hCG) at a dose of 250 micrograms (Merck Serono, Canada) or 10000 IU menopausal hCG (Fresenius Kabi Canada Ltd, Merck Serono Canada, Ferring Canada, Canada) or buserelyn 1000 IU (0.1 mg) (Sanofi Aventis Canada, Laval, Qc) was given for final oocyte maturation. 
Transvaginal ultrasound and rising serum estradiol (E2) levels were used to assess adequate follicular development, which was used to guide the timing of the hCG or leuprolide acetate injection.

Many of the cycles, particularly in the women 40 years of age or older, occurred in couples who refused donor sperm back up, had azoospermia and underwent testicular sperm aspiration (TESA) or microTESA, the day before oocyte collection per clinic protocol. These procedures failed to locate sperm and all oocytes were vitrified. The couple subsequently elected to use donor sperm and the oocytes were thawed and fertilized and transferred subsequently as fresh embryos obtained from frozen oocytes.

\section{Vitrification and warming of oocytes}

Vitrification and warming of mature oocytes were performed using a modification of the method described by Chian et al (11).

For vitrification, the oocytes were incubated in equilibration medium containing $7.5 \%$ (v/v) ethylene glycol (EG) and $7.5 \%(\mathrm{v} / \mathrm{v})$ dimethyl sulfoxide (DMSO) for 15 minutes, then transferred to vitrification medium containing $15 \%(\mathrm{v} / \mathrm{v})$ EG, 15\% (v/v) DMSO, and 0.5 M trehalose for one minute. The oocytes were then loaded onto a CryoTop (Kitazato Biopharma, Japan) and were immediately plunged into liquid nitrogen for storage.

For warming, the CryoTop was directly inserted into medium containing $1.0 \mathrm{M}$ trehalose for one minute at $37^{\circ} \mathrm{C}$. The oocytes were then transferred into diluent medium-I containing $0.5 \mathrm{M}$ trehalose for three minutes and then into diluent medium-II containing $0.25 \mathrm{M}$ trehalose for a further three minutes. Oocytes were washed twice in washing medium (three minutes each wash).

\section{Fertilization, culture}

Surviving oocytes were inseminated using intracytoplasmic sperm injection (ICSI) after completing the warming process. Oocytes were checked 18-20 hours post-ICSI for signs of fertilization. Embryos were cultured to the blastocyst stage in culture medium (Global total, Cooper surgical, USA). Embryo transfer was performed either on day 3 or day 5 . All embryos underwent laser assisted hatching using a ZILOS-tk (Hamilton Thorne Instruments Biosciences, Beverly, MA, USA) device to create an opening of approximately 20 microns in the zona pellucida.

\section{Endometrium preparation for Frozen embryo transfer}

Women were treated with estradiol valerate, $2 \mathrm{mg}$ orally three times daily, which was titrated up to $12 \mathrm{mg}$ daily as a combination of vaginal and oral intake. When the endometrium reached at least $8 \mathrm{~mm}$ in maximum anterior-posterior diameter measured trans-vaginally using a Vuluson 8 machine (GE, USA), vaginal progesterone was started the day prior to oocyte thawing and continued until 12 weeks of pregnancy, if pregnant.

\section{Statistical analysis}

Statistics were analyzed using SPSS, version 23.0 (IBM Inc., Chicago, IL, USA). Continuous data was assessed for normalcy using the Kolmogorov-Smirnov test. Data is presented as mean \pm standard deviation (SD) or percentage. Continuous data were analyzed using t-test and categorical data were analyzed using chi-squared testing. Non-parametric testing was used if indicated. A p-value $<0.05$ was considered to indicate statistical significance. Multivariate logistic regression was used to control for the confounding effects of female age and blastocyst or cleavage transfer. Power analysis suggested that with the current number of subjects, $82 \%$ power with alpha of $5 \%$ and effect size of $27 \%$ would be detectable.

\section{Results}

The study included 114 patients who underwent frozen embryo transfers. The patients were sub-divided into 67 (58.8\%) patients who underwent SET and 47 (41.2\%) who underwent DET. The mean age at the time of oocyte collection was $36 \pm 5$ years old for the 67 patients undergoing SET and $39 \pm 5$ years old for the 47 patients undergoing DET, which was significantly different $(p=0.001)$. Women in the study varied with $29.8 \%$ being under the age of 35 years old, $26.3 \%$ between the age of $35-40$ years old and $43.9 \%$ above the age of 40 years old at the time of oocyte collection (Table 1).

The mean $( \pm \mathrm{SD}$ ) overall survival rate of the oocytes was $83.5 \%$ ( \pm 19.7 ), while the mean ( \pm SD) overall survival of fertilized oocytes after ICSI was $70.1 \%$ ( \pm 22.4$)$. In regard to the maturation stage at transfer, $72 \%$ were cleavage embryos and $28 \%$ were blastocyst embryos. Among those who had cleavage stage embryos, 48.8\% underwent SET and 51.2\% underwent DET. Among patients in the blastocyst embryos group, $84.4 \%$ underwent SET and 15.6\% underwent DET (Table 1).

There were no statistically significant differences observed in pregnancy rates for SET $(38.1 \%)$ vs DET $(36.2 \%)(p=0.78)$ in all age groups (Table 2). Clinical pregnancy rates were similar between those undergoing SET (32.8\%) and DET (21.3\%) $(p=0.18)$. In relation to live birth rates, there were no statistically significant differences between the patients who underwent SET (28.4\%) and those who underwent DET (19.1\%) $(\mathrm{p}=0.26)$. Patients were divided into three different groups according to age: $(<35,35-40,>40$; Table 2$)$. In the $<35$ years old category, though there seems to be a relatively higher percentage of pregnancies ( $80 \%$ vs $48 \%$ ), clinical pregnancies ( $80 \%$ vs $34 \%$ ) and live births (60\% vs $34 \%$ ) in the DET group in comparison 
to the SET group, none of these values were statistically significant. In the 35 to 40 years old category, pregnancy (20\% vs $47 \%$ ), clinical pregnancy ( 0 vs $47 \%$ ) and live birth ( 0 vs $27 \%$ ) outcomes were similar between DET and SET groups, respectively. Furthermore, in the $>40$ year-old category, we also failed to demonstrate a statistically significant difference in pregnancy ( $37 \%$ vs $26 \%$ ), clinical pregnancy ( $22 \%$ vs $22 \%$ ) and live birth rates ( $22 \%$ vs $22 \%$ ) when patients who underwent DET as opposed to SET were compared $(\mathrm{p}=0.41, \mathrm{p}=0.68$, $\mathrm{p}=0.97$ ), respectively.

The multivariate multilevel analysis provided an adjusted odds ratio (95\% confidence interval) of 1.85 (0.46-7.44) for pregnancy, 0.497 (0.05-4.86) for clinical pregnancy, and 0.82 (0.11-6.29) for live birth when comparing SET and DET while controlling for age and stage of transfer, rendering the differences in outcomes between the two methods non-significant (Table 3).
Importantly, multiple gestation live birth rates were significantly lower in the SET (0\%), compared with DET group (44.4\%) $(p=0.01)$, in spite of the possible masked role of lower embryo quality or less favorable patient history in the DET group.

\section{Discussion}

The comparison between SET and DET in autologous frozen oocyte cycles as measured by rates of pregnancy, clinical pregnancy and live birth rates showed no differences in outcomes in women $<35,35-40$, and $>40$ years old. The use of DET, similar to other studies of frozen embryos, was again demonstrated to increase the risk of a multiple gestation (7-9). Our findings on the favourable use of SET while maintaining pregnancy success rates support previous literature that has addressed the same question (7-9). However, to the best of our

Table 1. Demographics and embryo characteristics of patients undergoing SET vs DET

\begin{tabular}{|c|c|c|c|c|c|}
\hline & & \multicolumn{2}{|c|}{ SET $(n=67)(58.8 \%)$} & DET $(n=47)(41.2 \%)$ & p-value $(95 \% \mathrm{CI})$ \\
\hline \multicolumn{2}{|c|}{ Age mean \pm SD (median) at time of oocyte collection } & \multicolumn{2}{|c|}{$36.45 \pm 5.2(35)$} & $39.64 \pm 5(41)$ & $0.001(-5.08 ;-1.24)$ \\
\hline \multirow{3}{*}{ Age at collection (stratified) } & $<35$ & \multicolumn{2}{|l|}{$29(43.3 \%)$} & $5(10.6 \%)$ & - \\
\hline & $35-39$ & \multicolumn{2}{|l|}{$15(22.4 \%)$} & $15(31.9 \%)$ & - \\
\hline & $>40$ & \multicolumn{2}{|l|}{$23(34.3 \%)$} & $27(57.4 \%)$ & - \\
\hline \multicolumn{2}{|c|}{$\begin{array}{l}\text { Cleavage stage embryo } \\
\text { Number of patients (\% of cleavage transfers) }\end{array}$} & \multicolumn{2}{|l|}{$40(48.8 \%)$} & $42(51.2 \%)$ & \multirow{2}{*}{$<0.001$} \\
\hline $\begin{array}{l}\text { Blastocyst stage embryo } \\
\text { Number of patients ( } \% \text { of bla }\end{array}$ & & \multicolumn{2}{|l|}{$27(84.4 \%)$} & $5(15.6 \%)$ & \\
\hline \multicolumn{6}{|l|}{ Age distribution $\mathrm{n}(\%)$} \\
\hline \multicolumn{3}{|l|}{$<35$} & \multicolumn{3}{|l|}{$34(29.8)$} \\
\hline \multicolumn{3}{|l|}{$35-39$} & \multicolumn{3}{|l|}{$30(26.3)$} \\
\hline \multicolumn{3}{|l|}{$>40$} & \multicolumn{3}{|l|}{$50(43.9)$} \\
\hline
\end{tabular}

Table 2. Pregnancy outcomes stratified by age at oocyte collection

\begin{tabular}{|c|c|c|c|c|}
\hline \multirow{5}{*}{ Pregnancy rate \% (number of pregnancy/no of transfers) } & Age & SET & DET & p-value \\
\hline & All & $38.1(26 / 67)$ & $36.2(17 / 47)$ & 0.78 \\
\hline & $<35$ & $48.3(14 / 29)$ & $80.0(4 / 5)$ & 0.32 \\
\hline & $35-39$ & $46.7(7 / 15)$ & $20.0(3 / 15)$ & 0.21 \\
\hline & $>40$ & $26.1(6 / 23)$ & $37.0(10 / 27)$ & 0.41 \\
\hline \multirow{4}{*}{ Clinical pregnancy rate $\%$ (number of clinical preg/no of transfers) } & All & $32.8(22 / 67)$ & $21.3(10 / 47)$ & 0.18 \\
\hline & $<35$ & $34.4(10 / 29)$ & $80.0(4 / 5)$ & 0.11 \\
\hline & $35-39$ & $46.7(7 / 15)$ & $0(0 / 15)$ & 0.03 \\
\hline & $>40$ & $21.7(5 / 23)$ & $22.2(6 / 27)$ & 0.68 \\
\hline \multirow{4}{*}{ Live birth rate \% (live birth /no of transfers) } & All & $28.4(19 / 67)$ & $19.1(9 / 47)$ & 0.26 \\
\hline & $<35$ & $34.4(10 / 29)$ & $60.0(3 / 5)$ & 0.39 \\
\hline & $35-39$ & $26.7(4 / 15)$ & $0(0 / 15)$ & 0.22 \\
\hline & $>40$ & $21.7(5 / 23)$ & $22.2(6 / 27)$ & 0.97 \\
\hline
\end{tabular}


Table 3. Unadjusted and adjusted odds ratios for reproductive outcomes. Single ET versus double ET

\begin{tabular}{|l|l|l|l|l|}
\hline Unadjusted & OR & $\mathbf{9 5 \%}$ CI & OR & $\mathbf{9 5 \% ~ C I ~}$ \\
\hline & 1.50 & $0.42-5.32$ & 1.85 & $0.46-7.44$ \\
\hline Pregnancy rate & 0.44 & $0.05-3.98$ & 0.497 & $0.05-4.86$ \\
\hline Clinical pregnancy & 0.71 & $0.10-5.03$ & 0.82 & $0.11-6.29$ \\
\hline Live birth rate & \begin{tabular}{l}
$|l|$ \\
\hline
\end{tabular} \\
ET: Embryo transfer, OR: Odds ratio, CI: Confidence interval. The odds \\
ratios adjusted for age and stage of embryos
\end{tabular}

knowledge there is limited data on women above the age of 40 years old when using vitrified oocytes. In fact, little is known about how oocytes from women 40 years of age or greater vitrify and the resultant pregnancy and live birth rates from these oocytes. Interestingly our live birth rates obtained in this study of approximately $22 \%$ in women who vitrified oocytes at 40 years of age are similar to our outcomes in women using fresh autologous oocytes at 41 and 42 years of age, which were $20 \%$ in 2018, without the use of PGT-A (9). In a recent scientific impact paper published by the British Royal College of Obstetricians and Gynaecologists on elective egg freezing for non-medical reasons, the authors stated that "Success rates will be limited in women who are already in their mid-late $30 \mathrm{~s}$ " (12). However; they could not cite a source for this statement. Our data suggests that success rates using autologous frozen oocytes remained similar to fresh IVF cycles when stratified for age although, they will be center specific. It should be noted that our success rates are preliminary and remain to be confirmed, given the relatively small number of subjects in our study. As such, we call on centers doing large numbers of cycles of elective oocyte cryo-preservation, particularly those in New York, to provide data stratified for age, so that success rates can be better established in this population.

Importantly, live birth rates after SET as a result of autologous oocyte vitrification was respectable at all age groups studied. However, bias must have existed in relation to allocation to SET or DET. Particularly, women with worse quality embryos or worse prognosis were more likely to receive DET in our study. Nevertheless, multiple gestation rates were high with DET in this group. Results suggest that SET is a viable and safe option after autologous oocyte vitrification, irrelevant of age.

Advanced maternal age (AMA) is generally well defined in the literature as maternal age above 35 years old. In Canada, the average age of mothers at first birth has been steadily increasing since the 1960s (13). Interestingly, Statistics Canada reported a shift in the age distribution of mothers who have a multiple birth (13). The proportion of women between the age of 35-39 years old who gave birth to twins increased from $9.8 \%$ to $23.1 \%$ in the span of 25 years and the proportion of those $40-44$ years old increased from $1.0 \%$ to $5.6 \%$. Simultaneously, there has been a decrease in the proportion of women who had twins and were in their late 20 s, from $38 \%$ to $24.4 \%$. The increased use of ART is the primary contributing factor to the increase in rates of twin births in older age groups. The results from our study suggest that SET is a viable alternative in women 40 years of age or greater when using vitrified oocytes. AMA is associated with fetal growth restriction, premature birth, neonatal intensive care unit admission, neonatal death, gestational diabetes, preeclampsia and stillbirth $(14,15)$. The combination of the plethora of inherent risks that accompany AMA pregnancies with the well-established risks associated with higher order pregnancies could lead to an overall increase in regard to maternal and fetal co-morbidities and should be avoided.

A factor commonly discussed in the literature regarding embryos transferred in a cycle is the stage or quality of the embryo. In our study, we observed that the SET group had an almost equal distribution of cleavage and blastocyst transfers, while the DET group had a majority of embryos in the cleavage stage (89.4\%) and fewer in the blastocyst stage (10.6\%). Overall, the literature presents heterogeneous findings regarding whether there is a benefit to transfer at one stage versus the other. A Cochrane systematic review, published in 2016, that reported on 27 randomized controlled trials and a total of 4,031 women found moderate quality evidence for clinical pregnancy when employing fresh blastocyst stage transfer (16). However, there was no difference found in cumulative clinical pregnancy rates when both fresh and thawed cycles from a single egg collection procedure were used. A subsequent systematic review and meta-analysis of reproductive outcomes including clinical pregnancy, live birth, ongoing pregnancy, cumulative pregnancy, and miscarriage, was published in May of 2017 by Martins et al. (17), in which the group reported on 12 randomized controlled trials and 1200 women, and found no evidence for the superiority of blastocyst compared to cleavage stage transfers. Further studies and meta-analyses are needed to assess how transfer of thawed embryos at the cleavage stage versus the blastocyst stage compare in terms of desired outcomes. In our study, even when adjusting for the stage of the embryo at the time of transfer, our results showed no differences in pregnancy, clinical pregnancy and live birth rates in the SET vs DET group over all. Further and larger studies are needed to confirm our results.

\section{Study limitation}

One of the limitations of our study was the small population size and non-randomization of the patient population. Given the nature of the trial, the employment of a randomized controlled trial may be difficult to implement. While one method of treatment is not inferior to another, randomizing 
patients to SET vs DET would put a group of patients at risk of a higher order pregnancy, which may not be a desired outcome of the woman or couple who are putting themselves through IVF treatment. In addition, we provide information on our outcomes of interest, including pregnancy, clinical pregnancy, live birth rates and multiple gestation live birth rates, but we did not further report on pregnancy complications observed or incidence of complications observed in the multiple gestation groups, which was unavailable at this time. The ages of some of the groups differed, although this was adjusted for using multivariate stepwise logistic regression analysis to control for confounding effects.

\section{Conclusion}

Vitrification of oocytes for women 35 years of age or older gives excellent pregnancy and live birth rates, similar to those seen with fresh autologous oocytes. This is even true for women of 40 years of age or greater. Multiple pregnancy rates are lowest with SET as opposed to DET, even after oocyte vitrification and even among women at least 40 years of age when the oocytes were vitrified. The limited use of DET, particularly in women over 35, may contribute to reduced rates of maternal and fetal co-morbidities by reducing the rate of multiple gestation live birth rates. Our study of 114 women does not support the use of DET in any age group, based on when oocytes were vitrified.

Ethics Committee Approval: McGill University Health Center Institutional Review Board (IRB) Ethics approval of this retrospective study was obtained (approval number: 20205631).

Informed Consent: Retrospective study.

Peer-review: Externally peer-reviewed.

Author Contributions: Surgical and Medical Practices: M.H.D., W.Y.S.; Concept: M.H.D., A.B.; Design: M.H.D., A.B.; Data Collection or Processing: A.B., R.K., S.A., W.Y.S.; Analysis or Interpretation: A.B.; Literature Search: A.B., M.H.D., W.Y.S.; Writing: A.B., R.K., S.A., W.Y.S., M.H.D.

Conflict of Interest: No conflict of interest is declared by the authors.

Financial Disclosure: The authors declared that this study received no financial support.

\section{References}

1. Bortoletto P, Bakkensen J, Anchan RM. Embryo transfer: timing and techniques. Minerva Endocrinol 2018; 43: 57-68.

2. Practice Committee of the American Society for Reproductive Medicine and the Practice Committee of the Society for Assisted Reproductive Technology. Criteria for number of embryos to transfer: a committee opinion. Fertil Steril 2013; 99: 44-6.

3. Multiple gestation pregnancy. The ESHRE Capri Workshop Group. Hum Reprod 2000; 15: 1856-64.

4. Martin JA, Hamilton BE, Osterman MJ. Three decades of twin births in the United States 1980-2009. NCHS Data Brief 2012; 1-8.

5. Aston KI, Peterson CM, Carrell DT. Monozygotic twinning associated with assisted reproductive technologies: a review. Reproduction 2008; 136: 377-86

6. Freeman MR, Hinds MS, Howard KG, Howard JM, Hill GA. Guidance for elective single-embryo transfer should be applied to frozen embryo transfer cycles. J Assist Reprod Genet 2019; 36: 939-46.

7. Tannus S, Son WY, Dahan MH. Elective single blastocyst transfer in advanced maternal age. J Assist Reprod Genet 2017; 34: 741-8.

8. Alasmari NM, Son WY, Dahan MH. The effect on pregnancy and multiples of transferring 1-3 embryos in women at least 40 years old. J Assist Reprod Genet 2016; 33: 1195-202.

9. Tannus S, Cohen Y, Son WY, Shavit T, Dahan MH. Cumulative live birth rate following elective single blastocyst transfer compared with double blastocyst transfer in women aged 40 years and over. Reprod Biomed Online 2017; 35: 733-8.

10. Dahan MH, Agdi M, Shehata M, Son W, Tan SL. A comparison of outcomes from in vitro fertilization cycles stimulated with either recombinant luteinizing hormone (LH) or human chorionic gonadotropin acting as an LH analogue delivered as human menopausal gonadotropins, in subjects with good or poor ovarian reserve: a retrospective analysis. Eur J Obstet Gynecol Reprod Biol 2014; 172: 70-3.

11. Chian RC, Huang JY, Gilbert L, Son WY, Holzer H, Cui SJ, et al. Obstetric outcomes following vitrification of in vitro and in vivo matured oocytes. Fertil Steril 2009; 91: 2391-8.

12. Anderson RA, Davies MC, Lavery SA; Royal College of Obstetricians and Gynaecologists. Elective Egg Freezing for Non-Medical Reasons: Scientific Impact Paper No. 63. BJOG 2020; 127: e113-21.

13. Provencher C, Milan A, Hallman S, D'Aoust C. Report on the Demographic Situation in Canada. 2016. https://www150. statcan.gc.ca/n1/en/pub/91-209-x/2018001/article/54956-eng. pdf?st $=$ zclCRGSe

14. Lean SC, Derricott H, Jones RL, Heazell AEP. Advanced maternal age and adverse pregnancy outcomes: A systematic review and meta-analysis. PLoS One 2017; 12(10): e0186287.

15. Pinheiro RL, Areia AL, Mota Pinto A, Donato H. Advanced Maternal Age: Adverse Outcomes of Pregnancy, A Meta-Analysis. Acta Med Port 2019; 32: 219-26.

16. Glujovsky D, Farquhar C, Quinteiro Retamar AM, Alvarez Sedo CR, Blake D. Cleavage stage versus blastocyst stage embryo transfer in assisted reproductive technology. Cochrane Database Syst Rev 2016; CD002118.

17. Martins WP, Nastri CO, Rienzi L, van der Poel SZ, Gracia C, Racowsky C. Blastocyst vs cleavage-stage embryo transfer: systematic review and meta-analysis of reproductive outcomes. Ultrasound Obstet Gynecol 2017; 49: 583-91. 